\title{
Speaker Tracking Using Multi-modal Fusion Framework
}

\author{
Anwar Saeed, Ayoub Al-Hamadi, and Michael Heuer \\ Institute for Electronics, Signal Processing and Communications (IESK), \\ Otto-von-Guericke-University Magdeburg, \\ D-39016 Magdeburg, P.O. Box 4210, Germany \\ \{Anwar. Saeed, Ayoub. Al-Hamadi\}@ovgu.de
}

\begin{abstract}
This paper introduces a framework by which multi-modal sensory data can be efficiently and meaningfully combined in the application of speaker tracking. This framework fuses together four different observation types taken from multi-modal sensors. The advantages of this fusion are that weak sensory data from either modality can be reinforced, and the presence of noise can be reduced. We propose a method of combining these modalities by employing a particle filter. This method offers satisfied real-time performance.
\end{abstract}

Keywords: Speaker tracking, Skin detection, Face detection, Particle filter, Time difference of arrival.

\section{Introduction}

This work represents an example of using multiple modalities within a particle filter. We then describe a particle filter by which multi-modal sensory data is fused together to track a speaker in a scene. For designing a particle filter for an application, it is necessary to introduce feedback to the system. This feedback should describe the scene. In the case of speaker tracking, we have two types of sensory input: video and audio input devices. Image processing methods operate on image sequence captured from the video input to detect the speaker and other approaches use the audio input for the speaker localization.

Tracking the speaker using audio-visual information is an active research topic in the computer vision due to its importance to various applications such as smart video-conferencing and surveillance security systems. Shivappa et al. [1] explored different strategies for audio-visual fusion. Vermaak et al. [2] described a method in which a standard contour tracking algorithm, consisting of an edge detector and a particle filter, is used in conjunction with a Time Difference of Arrival (TDOA) calculation to deduce the speaker location from auditory data received by a pair of microphones. The audio data are used for initialization and video data for localization in an attempt to utilize the strengths of each modality. This method enhances the existing visual tracking successfully and can detect speaker 'ping-pong'. However, this implementation is not a real-time solution. Zhou et al. 3. employed a histogram matching based technique for 
image based speaker detection and a TDOA algorithm once again for audio localization. The audio undergoes pre-processing to remove noise and a Kalman filter is used to further reduce spurious detections before both audio and video observations are passed to a Weighted Probabilistic Data Association (WPDA) filter for fusion and tracking. The aforementioned approach fused deferent types of sensory data; however, it did not provide real-time level of performance. In this paper, we address the speaker tracking with the help of audio-visual sensory data in real-time level.

We extract two observation data types from the video modality. The first one represents skin blobs that fit human face shape. The second observation type is the output of a human face detector. Additionally, two observation types are extracted from audio modality: the first one utilizes the time-difference of arrival of audio signal at two microphones, and the second one uses Received Signal Strength (RSS) to estimate the speaker location. These four observation types from the two modalities will then be combined in a useful and meaningful fashion using a particle filter and then used to detect and track the speaker in the scene.

The remainder of this paper is structured as follows. In section 2, we describe the video modality. Audio modality is explained in section 3 . Overview of the particle filter implementation is detailed in section 4. Experimental results are discussed in section 5. Finally, the conclusion and future perspectives are given in section 6 .

\section{Video Modality}

The sensor configuration used in this work is a mono-camera centered between two microphones. In the video modality, we segment the human face using two methods. The first one utilizes human skin color, while the second approach uses texture features.

\subsection{Face Detection Using Skin Color}

Several approaches were proposed to classify each pixel as skin or non-skin. Multivariate Gaussian mixture model (GMM) is an example of those approaches. Two GMMs could be built for skin and non-skin pixels. Then, each pixel with likelihood ratio exceeding an experimentally set threshold value is classified as skin [4]. However, this method is time-consuming in contrast to other methods that compare the pixel value with pre-learnt threshold values. This comparison could be carried out in different color spaces such as RGB, HSL, HSV, YCrCb, etc. 5]. In this paper, a combination of threshold filters in $\mathrm{HSV}$ and $\mathrm{YCrCb}$ is used to segment the skin pixels. The threshold values define boundaries for the pre-learnt skin color [6]. As we mentioned, we chose this skin detection technique due to its efficiency and to meet our requirement for building real-time speaker tracking approach. We chose chrominance channels $(c r, c b)$ from $\mathrm{YCrCb}$ color space and the Hue channel $(h)$ from HSV color space [6]. Each pixel $I(h, c r, c b)$ is classified as follows. 


$$
I(h, c r, c b)= \begin{cases}1 & H S V(h) \wedge C b C r(c r, c b) \\ 0 & \text { otherwise }\end{cases}
$$

where $H S V(h)$ represents the skin segmentation in HSV color space. The Hue component is proven to be a good discriminator for the human skin tone. $H S V(h)$ is calculated by

$$
H S V(h)=(h<25) \vee(h>230) .
$$

$\mathrm{CbCr}(\mathrm{cr}, \mathrm{cb})$ represents the skin segmentation in $\mathrm{YCrCb}$ color space. The luminance component $(\mathrm{Y})$ is ignored to have skin detection immune to the luminance variation. $\mathrm{CbCr}(\mathrm{cr}, \mathrm{cb})$ is calculated by

$$
C b C r(c r, c b)=\left\{\begin{array}{l}
(c r \leq 1.5862 \times c b+20) \wedge \\
(c r \geq 0.3448 \times c b+76.2069) \wedge \\
(c r \geq-4.5652 \times c b+234.5652) \wedge \\
(c r \leq-1.15 \times c b+301.75) \wedge \\
(c r \leq-2.2857 \times c b+432.85)
\end{array}\right\}
$$

At the end of skin pixel classification, we operate morphology operations to remove the outlier skin detection and to close misdetected pixels. The resulting skin regions are then examined for contours which may describe faces in the scene. The contours which do not fit the facial characteristics will be discarded.

\subsection{Face Detection Using Texture Features}

The face detected by skin color is error-prone due to cluttered environments and due to the existence of objects that are human skin colored with same face shape. Hence, we enhance the face detection by the use of texture features. This detection is more accurate; however, it detects the face only in a frontal upright pose with \pm 20 head rotation angles (yaw, pitch, and roll). Thus, the two methods of face detection will complement each other using the particle filter framework. We assign the detection using texture features more weight than that using skin color. We pass the same video frame, which is processed by skin segmentation, into a well-trained Haarcascade classifier [7/8. This classifier utilizes the Haarlike features, which are defined as the ratio of intensities of adjacent rectangles of different locations 9 .

\section{Audio Modality}

The audio data are processed by fast but rather imprecise Received Signal Strength RSS based location scheme and by much slower but far more accurate TDOA algorithm. Different precision and confidence factors are assigned to the locations estimated by TDOA and RSS according to the algorithms accuracy and reliability. 


\subsection{Audio TDOA}

It is possible to locate the origin of a sound source by observing the TDOA of signal at audio sensors placed in differing locations. The number of the used audio sensors determines the accuracy and dimensionality as well as the performance of the system. The processing using TDOA method is relatively costly, increasing exponentially with sensor count. To maintain real-time levels of performance, only two audio sensors are used in this implementation. This limits the output of the modality to a single dimension (the x-coordinate). However, as this dimension has the most variance, the chosen configuration gave an excellent balance of performance versus contribution. The algorithms used to estimate (TDOA) exploit the fact that the sound arriving at each microphone will be delayed according to the speaker position, given that the position of the microphones is known. To find this time delay, a cross-correlation function is used. Due to the nature of audio sensory information, multiple detections are possible for a single source. These ghost detections are the result of reverberations being interpreted falsely as signals originating directly from the source. To minimize the effect of these reverberations, we used a Generalized Cross Correlation function using a Phase Transform (GCC-PHAT), which was introduced by Knapp et al. [10]. This is a computationally expensive operation so the Discrete Fourier Transform (DFT) is used for efficiency. Given two signals $S_{1}$ and $S_{2}$, the weighted correlation function at time delay $\tau$ is

$$
G C C_{P H A T}(\tau)=\mathbf{F F T}^{-1}\left(\frac{F_{1}(f)\left[F_{2}(f)\right]^{*}}{\left|F_{1}(f)\left[F_{2}(f)\right]^{*}\right|}\right),
$$

where $F_{1}$ and $F_{2}$ are the Fourier transforms of $S_{1}$ and $S_{2}$, respectively. FFT ${ }^{-1}$ is the inverse Fourier Transform and [ ] ${ }^{*}$ denotes the complex conjugate. Only the frames containing speech will be processed. To determine the speech frames, we used Signal to Noise Ratio (SNR) along with Zero Crossing Rate [1].

\subsection{Audio RSS}

This Audio signal strength is far simpler than the previously described TDOA routine. However, in most cases it gives a decent approximation of the speaker position. It is not precise as TDOA. Consequently, this will be reflected in the particle filter parameters. Similar to TDOA, audio RSS is used to estimate the speaker position just in one dimension (x-coordinate). To measure audio RSS, the total energy is calculated for the signals contained in both left and right audio buffers. A signal contained in a buffer of size $N$ samples is defined as

$$
\mathbf{s}=(s(0), \ldots, s(N)) \text {. }
$$

Hence, the energy of a signal of size $N$ samples is

$$
e(\mathbf{s})=\sum_{n=0}^{N}[s(n)]^{2}
$$


By comparing the energy of each buffer, we can arrive at x-coordinate representing the relative position of a speaker in the scene. While being less precise, a greater confidence is placed in this Audio RSS compared to TDOA. This is due to the fact that RSS is less prone to noise such as reverberations.

The audio signals are continuously measured and buffered for (10ms-41ms) before using them for the location estimation by TDOA and RSS. Obviously, the buffers are cleared after each estimation, and the process iterates.

\section{Particle Filter Implementation}

Before discussing the fusion of multiple modalities, it is necessary to understand the means by which sensory data is incorporated into a particle filter. The points within a filter where sensory data is used are the measurement and selection stages. In the measurement stage, the particle set generated by the filter is examined and each particle is given a score, or weight, based on how accurately said the particle describes the scene. During the selection stage, the particle set is refined to accentuate those particles which best describe the scene, and new particles are introduced based on the current sensory data. The parameters of these steps vary on what the purpose of the filter is. In the case of the filter implementation given in this paper, it is intended that the particles should describe a bounding box relative to a camera snapshot showing where the current speaker is. In this case, each particle needs to be given some form of score based on how accurate the bounding box describes the real speaker position. The location of this real speaker from the raw sensory data and the choice of what weight to assign to each particle is our concern.

The general particle filtering scheme in 1213 is used in this work. The approach is particularly effective at tracking objects in substantial cluttered environments, which is very desirable when dealing with multiple modalities each potentially generates many observations and hence noise. The problem of speaker tracking can be formulated as a continues estimation of speaker state $\mathbf{x}_{t}$ at each time $t$. The state forms a vector containing a union of relevant parameters obtained from all modalities of feature extractors. The state $\mathbf{x}_{t}$ is defined here as

$$
\mathbf{x}_{t}=\left(x_{t}(1), x_{t}(2), x_{t}(3), x_{t}(4), x_{t}(5)\right) .
$$

$\left(x_{t}(1), \ldots, x_{t}(5)\right)$ denote $\mathrm{x}$-position, y-position, head width, head height, and head orientation angle, respectively. Particle Filters, a Quasi-Monte Carlo solution, solve the tracking issue. Let state $\mathbf{x}_{t}$ in the Markov state-space model represent a possible speaker configuration at time $t$. And $\mathbf{y}_{t}$ is the observation obtained using the two modalities, as discussed in sections (3] and 2). Then, the distribution $p\left(\mathbf{x}_{t} \mid \mathbf{y}_{1: t}\right)$ can be calculated by

$$
p\left(\mathbf{x}_{t} \mid \mathbf{y}_{1: t}\right) \propto p\left(\mathbf{y}_{t} \mid \mathbf{x}_{t}\right) \int_{\mathbf{x}_{t-1}} p\left(\mathbf{x}_{t} \mid \mathbf{x}_{t-1}\right) p\left(\mathbf{x}_{t-1} \mid \mathbf{y}_{1: t-1}\right) \mathrm{d} \mathbf{x}_{t-1}
$$

Eq. 7 consists of likelihood function $p\left(\mathbf{y}_{t} \mid \mathbf{x}_{t}\right)$ multiplied by an integral representing the prediction step. The particle filtering method used here is an 
approximation to Eq.77. At each time $t$, there are $N$ samples $\left\{\mathbf{x}_{t}^{(i)}, \quad i=1, \ldots, N\right\}$ each associated with weight value $w_{t}^{(i)}$. To avoid the degeneracy problem caused in re-sampling, the weight value is calculated as follows [14].

$$
w_{t}^{(i)} \propto p\left(\mathbf{y}_{t} \mid \mathbf{x}_{t}^{(i)}\right) .
$$

In the particle prediction step, the state of each particle is altered according to an underlying temporal model, as given in Eq. 9.

$$
\mathbf{x}_{t}=\mathbf{A} \mathbf{x}_{t-1}+\mathbf{B} \mathbf{n}_{t} .
$$

Where $\mathbf{A}$ and $\mathbf{B}$ are experimentally set parameters for the model. $\mathbf{n}$ is a normalized white Gaussian noise vector. Within the update step, we assign each sample $\mathbf{x}_{t}^{(i)}$ new weight value according to the observation likelihood as in Eq. 8. Where the observation likelihood function is defined as an averaged sum of likelihood functions of the particle components,

$$
p\left(\mathbf{y}_{t} \mid \mathbf{x}_{t}\right)=\frac{1}{5} \sum_{i=1}^{5} p\left(\mathbf{y}_{t} \mid x_{t}(i)\right)
$$

As we mentioned in sections (3) and 2), we have four different observation types. In addition, we could have multiple observations from each type. For example, more than one face could be detected and may be many locations could be estimated for the speaker in each observation type. The component $x(1)$ is fused from the four observation types, while the other four components are fused only from the video modality. Each component $x(i)$ has precision factors $\sigma_{i, m}$ and confidence factors $\gamma_{i, m}$ reflecting the observation type $(m)$ accuracy and reliability, respectively. Let us have $Z$ observations for the component $x_{t}(i)$. These observations are denoted by $\left(y_{t, 1}^{m}(i), \ldots, y_{t, Z}^{m}(i)\right)$, where $m$ denotes the observation type. Then, we formulate the observation likelihood function for each component as GMM given by

$$
p\left(\mathbf{y}_{t} \mid x_{t}(i)\right)=K \sum_{o=1}^{Z} \frac{\gamma_{i, m}}{\sqrt{2 \pi \sigma_{i, m}^{2}}} \exp \left(-\frac{\left(y_{t, o}^{m}(i)-x_{t}(i)\right)^{2}}{2 \sigma_{i, m}^{2}}\right),
$$

where $K$ is a normalization factor, which depends on the observation number $Z$. Obviously, $K$ is the reciprocal of the sum of the confidence factors $\gamma_{i, m}$ for all observations. Finally, the density distribution $p\left(\mathbf{x}_{t} \mid \mathbf{y}_{1: t}\right)$ in Eq. 7 is approximated by a sum of $N$ Dirac functions as follows.

$$
p\left(\mathbf{x}_{t} \mid \mathbf{y}_{1: t}\right) \approx \sum_{i=1}^{N} w^{(i)} \delta\left(\mathbf{x}_{t}-\mathbf{x}_{t}^{(i)}\right)
$$

\section{Experimental Results}

The performance of the particle filter is more than sufficient for real-time operation. This filter is employing the four input data, as described in sections (2) and 3 ). 
An internal timer of the application iterates the filter every $10 \mathrm{~ms}$, which is enough for most iterations; however, when a slowdown occurs due to the presence of more observations, it has never been significant enough to go beyond the 24 frames per second ( $41 \mathrm{~ms}$ per iteration) limit. The main observable distinction between the multi-modal approach as opposed to single modal one is that the system is much more robust when dealing with noise or non detection. For example, the Haarlike face detection observations are not always present due to the orientation of speaker face in the scene; however, both the audio and skin color modalities allow tracking to continue. Conversely, over abundance of observations generated by the skin color segmentation is complemented by the accuracy Haar-like face detection meaning that the tracker does not get distracted by the multiple false positives. Fig. 1 (a,b) shows two samples of our experiment. The two images are overlaid with data extracted from the audio and video modalities. The probability density distribution of $\mathrm{x}$-component is given below each image. The speaker was detected and tracked successfully.

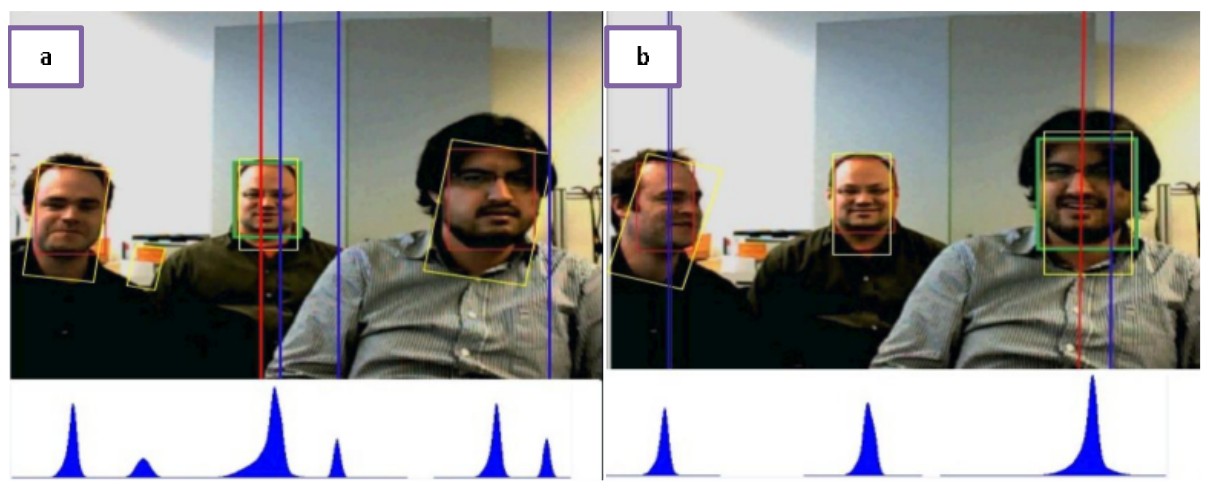

Fig. 1. Images showing the particle filter performance. The red and yellow rectangles show measurements from the video modality and the vertical blue and red lines from the audio modality. Below each image is the probability density distributions for the $\mathrm{x}$-component given the measurements shown. The green rectangle is the filter output corresponding to the greatest peak in the probability distribution. In (a), the center person is speaking whereas in (b) the right person is doing the majority of the speaking with some sound coming from the left.

\section{Conclusions and Future Work}

We have shown an approach for speaker tracking. We combined four observation types from two modalities (audio-visual). Each observation could not be used alone; however, when we combined them within a particle filter, we achieved a robust performance. The observations are modeled by GMMs with experimentally set parameters. Our proposed approach is able to perform in real time on standard workstation. In future, we will consider adding more microphones. Hence, we will be able to estimate the speaker location in y-coordinate as well. 
Acknowledgments. This work is supported by Transregional Collaborative Research Centre SFB/TRR 62 "Companion-Technology for Cognitive Technical Systems" funded by DFG and OvG-University Magdeburg.

\section{References}

1. Shivappa, S., Trivedi, M., Rao, B.: Audiovisual information fusion in human computer interfaces and intelligent environments: A survey. Proceedings of the IEEE 98, 1692-1715 (2010)

2. Vermaak, J., Gangnet, M., Blake, A., Perez, P.: Sequential monte carlo fusion of sound and vision for speaker tracking. In: ICCV, pp. 741-746 (2001)

3. Zhou, H., Taj, M., Cavallaro, A.: Target detection and tracking with heterogeneous sensors. IEEE Journal of Selected Topics in Signal Processing 2, 503-513 (2008)

4. Saeed, A., Niese, R., Al-Hamadi, A., Michaelis, B.: Coping with hand-hand overlapping in bimanual movements. In: 2011 IEEE International Conference on Signal and Image Processing Applications (ICSIPA), pp. 238-243 (2011)

5. Schettini, R., Gasparini, F.: Skin segmentation using multiple thresholding. In: Internet Imaging VII, IS and T/SPIE, pp. 60610F-1-60610F-8. SPIE (2006)

6. Rahman, N.A., Wei, K.C., See, J.: RGB-H-CbCr Skin Colour Model for Human Face Detection. In: Proceedings of The MMU International Symposium on Information \& Communications Technologies, M2USIC 2006 (2006)

7. Saeed, A., Niese, R., Al-Hamadi, A., Panning, A.: Hand-face-touch measure: a cue for human behavior analysis. In: 2011 IEEE International Conference on Intelligent Computing and Intelligent Systems (ICIS), vol. 3, pp. 605-609 (2011)

8. Bradski, G.: The OpenCV Library. Dr. Dobb's Journal of Software Tools (2000)

9. Viola, P., Jones, M.: Rapid object detection using a boosted cascade of simple features, pp. 511-518 (2001)

10. Knapp, C.H., Carter, G.C.: The generalized correlation method for estimation of time delay. IEEE Trans. Acoust., Speech, Signal Processing 24, 320-327 (1976)

11. Bachu, R.G., Kopparthi, S., Adapa, B., Barkana, B.D.: Separation of voiced and unvoiced using zero crossing rate and energy of the speech signal. In: American Society for Engineering Education ASEE Zone Conference Proceedings, pp. 1-7 (2008)

12. Blake, A., Isard, M.: The CONDENSATION algorithm - conditional density propagation and applications to visual tracking. In: NIPS, pp. 361-367 (1996)

13. Steer, M., Al-Hamadi, A., Michaelis, B.: Audio-visual data fusion using a particle filter in the application of face recognition. In: 2010 20th International Conference on Pattern Recognition (ICPR), pp. 4392-4395 (2010)

14. Doucet, A., De Freitas, N., Gordon, N. (eds.): Sequential Monte Carlo methods in practice (2001) 